\title{
Estrus- and Steroid-Induced Changes in Circadian Rhythms in a Diurnal Rodent, Octodon degus
}

\author{
SUSAN E. LABYAK* AND THERESA M. LEE ${ }^{*}$ \\ Departments of $*$ Nursing and $†$ Psychology, 1103 E. Huron, Neuroscience Laboratory Building, University of \\ Michigan, Ann Arbor, MI 48104-1687, E-Mail: terrilee@UMICH.edu
}

Received 2 August 1993

\begin{abstract}
LABYAK, S. E. AND T. M. LEE. Estrus and steroid-induced changes in circadian rhythms in a diurnal rodent, Octodon degus. PHYSIOL BEHAV 58(3) 573-585, 1995. - Diurnal Octodon degus exhibited marked alterations in activity and temperature in conjunction with the $3 \mathrm{wk}$ estrous cycle when housed in LD12:12 light cycle. On the day of estrus, mean daily activity increases $109 \%$, mean core temperature rises $.4^{\circ} \mathrm{C}$, activity onset is advanced $2 \mathrm{~h}$, and amplitudes of both rhythms decline compared with the 3 days prior to estrus. On the day following estrus, activity onset was delayed $4.9 \mathrm{~h}$, and mean activity and core temperature fell below that of the preestrus period. Ovariectomy significantly reduced mean temperature $\left(.98^{\circ} \mathrm{C}\right)$ but did not significantly alter mean activity, and eliminated cyclic effects of estrus. Estrogen replacement led to a nonsignificant elevation in mean activity and core temperature with no change in the phase angle of entrainment. Progesterone replacement significantly reduced mean core temperature and mean activity, while only the phase angle difference between temperature minimum and activity onset was significantly altered. Intact degus maintained in constant darkness displayed only transient fluctuations in activity onset and temperature minimum during and after estrus. Estrogen or progesterone treatment of ovariectomized, free-running degus altered mean temperature and activity levels, but did not influence tau. Changes in phase angle of entrainment during estrus are not the result of hormone effects on the circadian clock but likely reflect increased or decreased levels of activity.
\end{abstract}

Octodon degus Estrus Circadian Activity Temperature Ovariectomy Free-running rhythm

Phase angle of entrainment

OVARIAN hormones influence the timing and expression of circadian activity and core temperature rhythms in nocturnal female rodents $(1,13,15,31)$. During proestrus and estrus (days three and four of a four-day estrous cycle) when estrogen levels are high (4), hamsters demonstrate an increase in mean daily activity (25), a phase advance in the time of activity onset $(22,24)$, and a rise in mean core temperature (24). In blind, ovariectomized female hamsters estradiol shortened the free-running circadian activity period $(21,22)$, and this effect could be blocked by the administration of progesterone (31). These findings suggest that estradiol and progesterone interact to modulate the timing and expression of circadian activity in the hamster.

Rats display similar estrous-related changes in circadian activity (26) and core temperature $(20,35)$ in their five-day estrous cycle. On the day of estrus, total activity and activity duration increase in Sherman and LEW/ZTM rats. Activity onset is phase advanced and the circadian period is notably shortened in both entrained and free-running animals $(1,20,35)$. In other strains of rats (e.g., Holtzman and Sprague-Dawley), increased runningwheel activity and temperature elevations occur on the night of proestrus $(2,15,37)$, accompanied by a phase advance in the circadian activity and core temperature rhythms. In contrast to studies in which animals were housed with running-wheels, Kent et al. (15) found that sedentary Sprague-Dawley rats, as well as those housed in cages with locked wheels, displayed a significant phase delay in the temperature rhythm on each day of the estrous cycle. They concluded that estrous-related changes in core temperature were largely a function of increased activity, and that ovarian hormones had little influence on temperature in SpragueDawley rats.

While an estrous effect on circadian activity and core temperature rhythms has been documented in nocturnal rodents, few investigators have studied the impact of estrus on circadian rhythms in diurnal mammals. Since species-typical patterns are evident in nocturnal species, estrous-related alterations in circadian rhythms may be distinctly different in diurnal species. For example, in one case study, the circadian activity of a premenopausal 46 yr old woman was monitored for one year via wristactograph. At the time of ovulation, the subject demonstrated a significant decrease in mean activity coupled with a significant phase delay in activity onset (7). The findings suggest that circadian responsiveness to changing ovarian hormones is distinctly different in diurnal humans when compared to nocturnal rats or hamsters. Wever (34) studied changes in the free-running core temperature rhythm across the menstrual cycle in young women, and compared them to mean temperatures in men of similar ages. While preovulatory mean temperatures in young women were similar to those of males, postovulatory mean temperatures were

\footnotetext{
${ }^{1}$ To whom requests for reprints should be addressed.
} 
higher. Such postovulatory temperature changes have not been reported for hamsters and rats, and may be the result of nocturnal/ diurnal differences or the difference in length of the luteal stage.

Diurnal female degus (Octodon degus) display altered circadian activity and core temperature rhythms at approximately 20 day intervals in this laboratory. The purpose of these experiments was to determine whether periodic circadian changes were the result of estrus, and if so, to determine the potential role of estrogen and progesterone in these changes. The daily mean, phase, and amplitude of the circadian activity and core temperature rhythms were monitored. If data from diurnal degus are consistent with those from nocturnal rodents then we would expect that (i) female degus would demonstrate a phase advance in circadian activity and core temperature rhythms during proestrus and estrus, coupled with an increase in mean daily activity and core temperature; (ii) mean core temperature during proestrus/estrus might rise in conjunction with increased mean activity; (iii) ovariectomy would eliminate the cyclic alterations in circadian activity and core temperature associated with estrus; and (iv) estrus or replacement hormones would influence $\tau$ consistent with hormone-induced changes in entrained phase.

\section{METHODS}

\section{Animals and Housing}

Fourteen young, adult female degus (6-16 mo.; average lifespan 5-7 yr) reared in a laboratory colony at the University of Michigan were used in these experiments $(N=5-10$ per experiment). Parental stock was derived from colonies in five locations in the U.S., and outbreeding was used to maintain genetic diversity in the population. The degus were individually housed in $47.5 \times 26.25 \times 20 \mathrm{~cm}$ cages fitted with running-wheels $(9 \mathrm{~cm}$ wide and $34.5 \mathrm{~cm}$ in diameter). Food and water were available ad lib, and room temperature was maintained at $22^{\circ} \pm 2^{\circ} \mathrm{C}$. Degus were maintained in $12 \mathrm{~h}$ of light per day (LD 12:12; lights on at $0600 \mathrm{~h}$ EST) for 3 wk prior to beginning data collection.

\section{Surgery}

The degus were implanted intraperitoneally with Mini-mitter transmitters (model VM-FH; Mini-mitter, Inc., Sun River, OR) to measure core temperature and general activity with Dataquest III hardware and software. Degus were anesthetized with Ketamine HCL ( $30 \mathrm{mg} / \mathrm{kg}$ of body weight) and Xylazene $(2.5 \mathrm{mg} / \mathrm{kg}$ of body weight). The animals required approximately three hours to recover, and Yohimbine ( $2.5 \mathrm{mg} / \mathrm{kg}$ of body weight, IP) was administered immediately following the surgery to shorten the recovery period.

\section{Data Collection and Analysis}

The animals were given at least 1 wk to recover prior to experimentation. Running-wheel activity was recorded as the number of wheel revolutions and was stored at $10 \mathrm{~min}$ intervals by Dataquest. General activity and core temperature were monitored via Mini-mitter transmitters at $10 \mathrm{~min}$ intervals. The estrous cycle was monitored daily by microscopic examination of washes from vaginal lavage (11).

Spectral analysis and Chi square periodograms were used to verify the $24 \mathrm{~h}$ period of the entrained activity and core temperature rhythms (10). The Tau and Dataquest III computer software programs (Mini-Mitter, Inc., Sunriver, OR) were used for data display (actograms and frequency histograms) and analysis. Repeated measures ANOVA tests were used to compare activity and core temperature across different stages of the estrous cycle with posthoc paired comparisons used to test differences between specific stages of the estrous cycle. Summary statistics are reported as the mean + SEM, and differences considered significant if $p<0.05$.

Four parameters for the activity and core temperature rhythms were examined: the daily mean, the phase angle of entrainment of activity onset $\left(\Psi_{\mathrm{AO}}\right)$ and temperature minimum $\left(\Psi_{\mathrm{TM}}\right)$ with the timing of lights-on, the phase angle of entrainment of the first morning peak of activity $\left(\Psi_{\mathrm{AP}}\right)$ and morning peak in temperature $\left(\Psi_{\mathrm{TP}}\right)$ with the timing of lights-on, and the daily rhythm amplitude (from mean to peak value). The time of activity onset and temperature minimum were determined by examining frequency histograms (Fig. 1). Activity onset was defined as the time when an animal first demonstrated a minimum of 40 counts of activity in a $10 \mathrm{~min}$ interval, and was active for at least four consecutive 10 min intervals. Temperature minimum was defined as the time of the lowest daily temperature readings (for three to four consecutive $10 \mathrm{~min}$ intervals). $\Psi_{\mathrm{AO}}$ and $\Psi_{\mathrm{TM}}$ were derived by calculating the difference between the time of lights-on and the time of activity onset and temperature minimum, respectively. $\Psi_{\mathrm{AP}}$ and $\Psi_{\mathrm{TP}}$ were derived by calculating the difference between the time of lights-on and the time of the morning peak in activity and core temperature, respectively (Fig. 1). The phase angle relationship between temperature minimum and activity onset $\left(\Psi_{\mathrm{TA}}\right)$, calculated by $\Psi_{\mathrm{TM}}-\Psi_{\mathrm{AO} \text {, }}$, was also compared across the estrous cycle. Rhythm amplitudes were determined by calculating the difference between the rhythm peak (maximum value) and the rhythm mean, where the $24 \mathrm{~h}$ mean was computed from midnight each day.

\section{EXPERIMENT ।}

Twenty-one estrous cycles from ten degus (i.e., each animal contributing two or three estrous cycles to the data base) were used to examine the effect of estrus on the circadian activity and core temperature rhythms. Data were analyzed in four periods: (i) preestrus consisted of the three days preceding estrus; (ii) estrus consisted of one to two days; (iii) metestrus consisted of the day immediately following estrus; and (iv) postestrus consisted of the two days following metestrus. The data were similar across animals and were therefore pooled.

\section{Results}

The animals displayed stable entrainment under LD 12:12 and demonstrated vaginal changes indicating estrous cycles of 20.5 \pm .5 days. Vaginal lavage could only be carried out for three to five days per cycle; during the remainder of the cycle the vaginae were not patent. While daily vaginal lavage distinguished nonestrous from proestrous and estrous days, proestrus and estrus could not always be distinguished because the animals were often in transition between these stages at the time of lavage.

Estrous smears correlated with a marked rise in mean daily activity and core temperature (Figs. 2 and 3). The animals demonstrated a significant increase in mean running-wheel activity (109\% increase; $p<0.001$ ) from preestrus to the day of estrus (Fig. 3A). This was followed by a significant decline in mean activity on the day of metestrus (67\% decrease from estrus; $p<$ 0.001 ), and recovery during the postestrous period to preestrous baseline. Mean changes in activity across the estrous cycle were not the result of changes in daily peak activity levels.

On the day of estrus, there was a $1.9 \mathrm{~h}$ advance in $\Psi_{\mathrm{AO}}(p<$ 0.007 ; Table 1 ), followed by a $4.9 \mathrm{~h}$ delay $(p<0.001$; Table 1$)$ on the day of metestrus, and a return to baseline preestrous state during the postestrous period. $\Psi_{\mathrm{AP}}$ was advanced $1.5 \mathrm{~h}(p=$ 0.055 ; Table 1) on the day of estrus, and delayed $3.5 \mathrm{~h}$ during metestrus, although this shift did not reach significance (Table 1). In conjunction with the advance in $\Psi_{\mathrm{AO}}$ and $\Psi_{\mathrm{AP}}$, the ampli- 


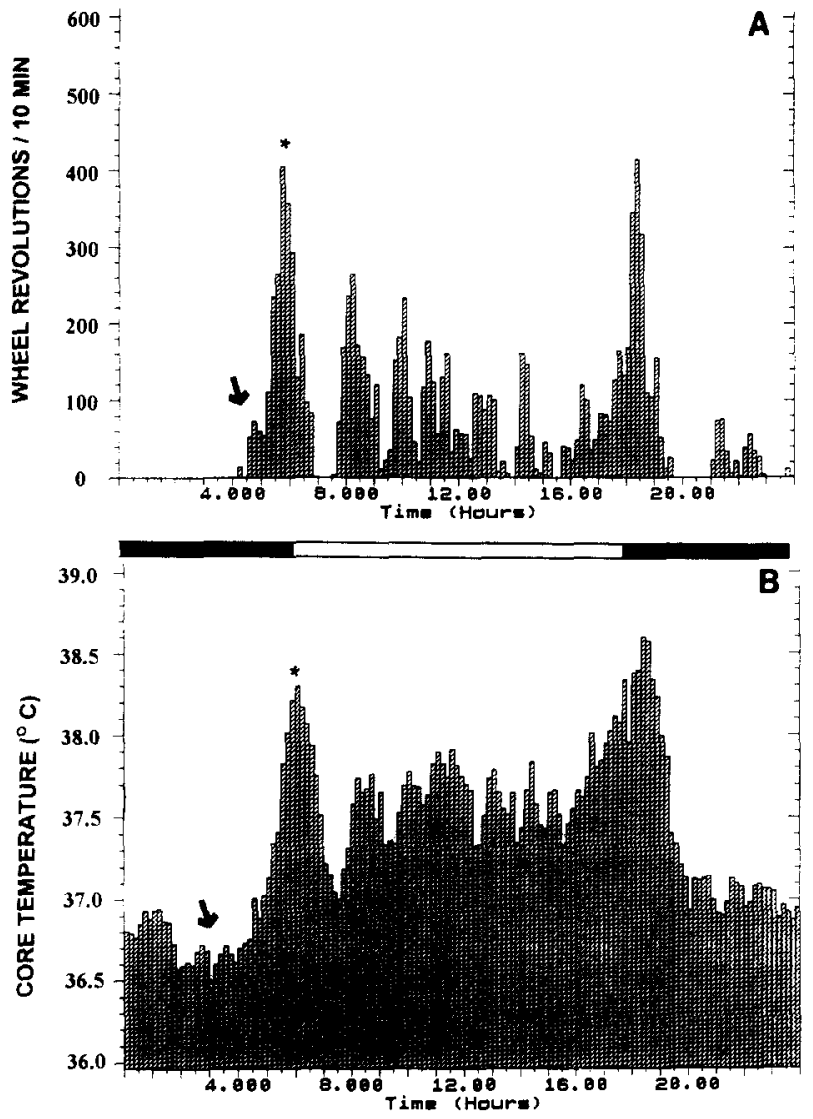

FIG. 1. Frequency histograms of one day of (A) running-wheel activity and $(B)$ core temperature for a single female degu. The arrows indicate phase reference points for activity onset $\left(\Psi_{\mathrm{AO}}\right)$ and temperature minimum $\left(\Psi_{\mathrm{TM}}\right)$. The asterisks indicate the phase reference points for first activity ( $\left.\Psi_{\mathrm{AP}}\right)$ and temperature $\left(\Psi_{\mathrm{TP}}\right)$ peaks after midnight. The bar in the center of the graph indicates the light:dark schedule.

tude of the circadian activity rhythm declined $11.3 \%$ during estrus $(p<0.008 ;$ Fig. $4 \mathrm{~A})$, but did not differ from baseline during metestrus when $\Psi_{\mathrm{AO}}$ was significantly delayed. The decline in amplitude during estrus was due to the increased duration of elevated activity.

Mean daily core temperature rose $.4^{\circ} \mathrm{C}$ during estrus $(p<$ $0.001 ; \mathrm{Fig} .3 \mathrm{~B})$, and then declined $.66^{\circ} \mathrm{C}$ from estrus to metestrus $(p<0.001)$. While there was no significant change in the daily minimum temperature across the estrous cycle, temperature maximum declined significantly between preestrus or estrus and metestrus ( .4 and $.5^{\circ} \mathrm{C} ; p<0.03$ and $p<0.001$, respectively).

In contrast to the advance observed in the $\Psi_{\mathrm{AO}}$ and $\Psi_{\mathrm{AP}}$ on the day of estrus, there was a nonsignificant $.59 \mathrm{~h}$ advance in $\Psi_{\mathrm{TM}}$ on the day of estrus. The changes in $\Psi_{\mathrm{TP}}$ across the estrous cycle approached significance (2-tailed posthoc tests of significance); temperature peak advanced $1.2 \mathrm{~h}$ on the day of estrus $(p=0.065)$ and delayed $3.5 \mathrm{~h}$ between estrus and metestrus $(p=0.075$; see Table 1). The amplitude of the circadian temperature rhythm declined $16.4 \%$ between preestrus and estrus $(p<0.02$; Fig. 4B). The decline in rhythm amplitude during estrus was due to an increase in the duration of elevated temperatures.

Thus, on the day of estrus mean activity and core temperature rose as the amplitude of those rhythms declined. In addition, the onset of activity $\left(\Psi_{\mathrm{AO}}\right)$ and the first morning peak of activity $\left(\Psi_{\mathrm{AP}}\right)$ were phase advanced on the day of estrus. Metestrus was marked by a reduction in mean activity and core temperature, and a phase delay in $\Psi_{\mathrm{AO}}$. Recovery of the temperature amplitude during metestrus and postestrus was gradual, while the amplitude of the activity rhythm returned to baseline during metestrus. The phase angle difference between temperature minimum and activity onset $\left(\Psi_{T A}\right)$ was significantly altered during estrus. $\Psi_{T A}$ was reduced by $1.6 \mathrm{~h}$ from preestrus to estrus $(p<0.03)$, but increased to 4 $\mathrm{h}$ during metestrus $(p<0.005)$ before returning to the preestrous relationship (Table 1). The alteration in $\Psi_{T A}$ was primarily due to changes in $\Psi_{\mathrm{AO}}$, while $\Psi_{\mathrm{TM}}$ remained essentially constant.

\section{EXPERIMENT 2}

Circadian activity and core temperature rhythms in the degu are tightly coupled. Activity onset begins as temperature rises, and activity/temperature elevations throughout the day are strikingly similar. This strong association between circadian activity and core temperature rhythms makes it difficult to evaluate the effects of ovarian hormones on the temperature rhythm of intact animals with running-wheels. Increases in mean activity correlate well with temperature elevations. However, mean temperature

\section{A}

B

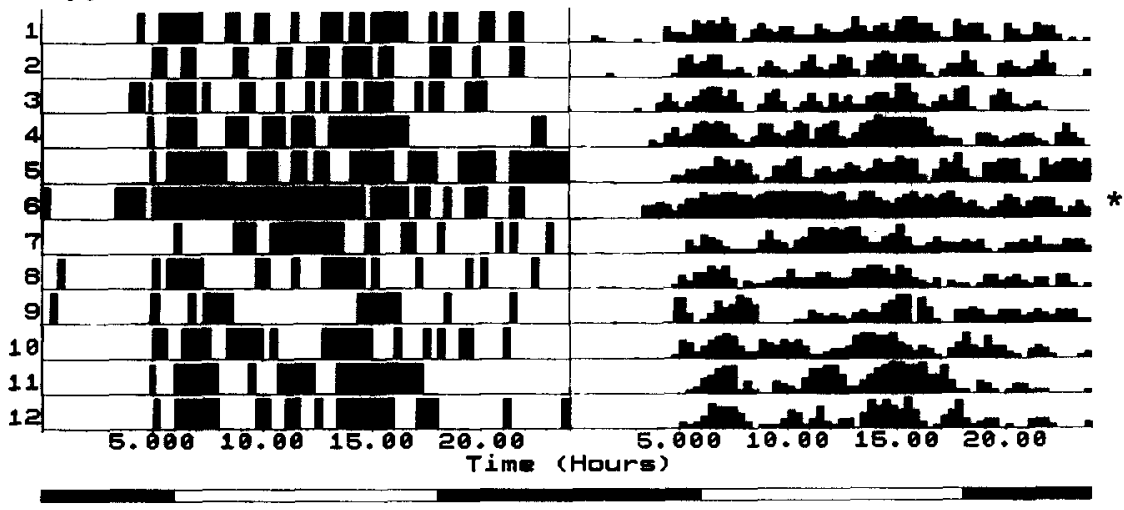

FIG. 2. Entrained animal's (A) activity and (B) core temperature actograms during preestrus, estrus and postestrus changes. Each line represents one day of data. Asterisk denotes the day of estrus. Bar at the bottom of the actograph indicates the light:dark schedule. The lowest $25 \%$ of temperature data are not displayed to assist in viewing the daily rise in temperature. 


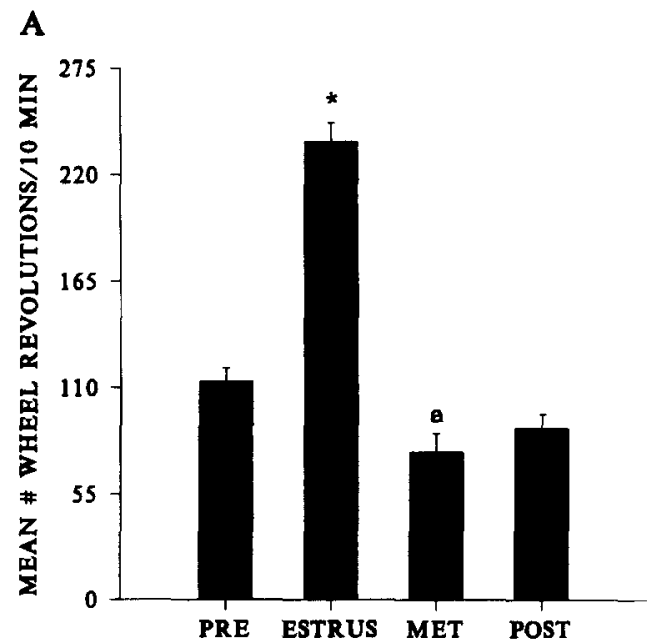

B

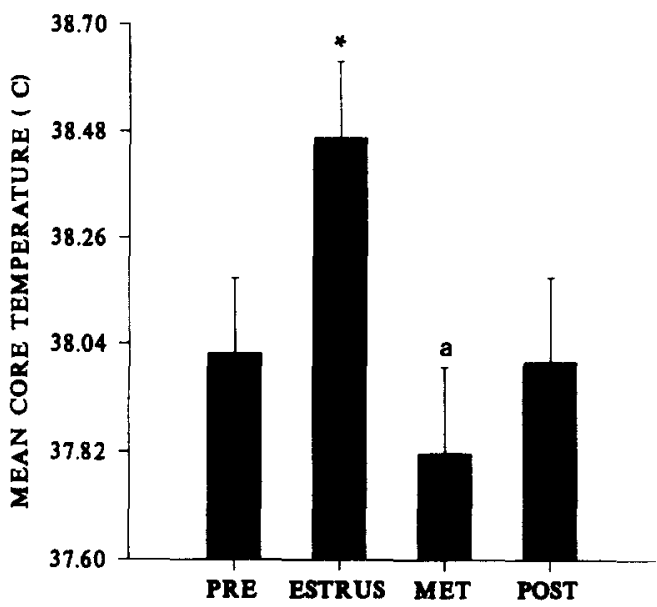

FIG. 3. Variations in mean ( \pm SEM) (A) running-wheel activity and (B) core temperature across the estrous cycle. Based on vaginal lavage, PRE $=3$ days preestrus, ESTRUS $=24$ to $36 \mathrm{~h}$ around estrus, MET $=24 \mathrm{~h}$ following estrus, and POST $=2$ days following metestrus. All days begin at midnight. Asterisks represent significant differences from preestrus using posthoc paired comparisons $(p<0.001)$. Letter " $a$ " represents significant differences between estrus and metestrus with posthoc paired comparisons $(p<0.001)$.

can rise without increasing activity, although this has not been reported in other rodents unless they have fevers $(15,24,28)$. To further evaluate the influence of running-wheel activity on core temperature, the mean total activity (gathered by the Mini-mitter/ Dataquest system) and core temperature of seven female degus (6-8 mo old), with and without running-wheels available, were examined across two or three estrous cycles. Effects of the estrous cycle on the circadian activity and core temperature rhythms were evaluated across the four periods described in Experiment 1: preestrus, estrus, metestrus, and postestrus. Repeated measures ANOVA tests with posthoc paired comparisons were used to examine differences between the running-wheel and nonrunning-wheel treatments, and differences were considered significant if $p<0.05$.

\section{Results}

During the time that animals had access to running-wheels, they demonstrated a significant rise in general activity during estrus ( $p<0.001$; Fig. 5A), followed by a significant decline in activity from estrus to metestrus $(p<0.001)$ which continued into the postestrous period $(p<0.001)$. Mean core temperature also rose between preestrus and estrus ( $p<0.001$; Fig. 5B), and fell significantly between estrus and metestrus $(p<0.001)$ and between estrus and postestrus $(p<0.001)$.

Although total activity (includes activity generated while in a running wheel) was considerably diminished $(54 \%, p<0.001)$ when running-wheels were removed, the animals without running-wheels continued to demonstrate a significant rise in activity during estrus ( $p<0.05$; Fig. 5A). The percent change in mean daily activity on the day of estrus was comparable with or without a running-wheel (54\% and $40.4 \%$, respectively). Activity declined between estrus and metestrus $(28.9 \% ; p<0.05)$, and remained lower during the postestrous period $(p<0.05)$. Although mean core temperature declined $.32^{\circ} \mathrm{C}$ following removal of the wheel $(p<0.001$; Fig 5B), the degus continued to display a significant $.4^{\circ} \mathrm{C}$ rise in core temperature during estrus $(p<0.02)$, a significant $.4^{\circ} \mathrm{C}$ decline between estrus and metestrus $(p<$ $0.001)$, and a $.55^{\circ} \mathrm{C}$ decline between estrus and postestrus $(p<$ 0.01 ; Fig. 5B). Again, the percent change in core temperature on the day of estrus was comparable with or without running-wheel activity $(1.14 \%$ and $1.19 \%$, respectively). Thus, the heavy activity associated with the running-wheel was not responsible for estrous-related elevations in mean activity and core temperature.

\section{EXPERIMENT 3}

The influence of estrogen and progesterone on circadian activity and core temperature rhythms was evaluated by first eliminating and then restoring those hormones. Nine female degus from Experiment 1 were anesthetized as previously described, and bilateral ovariectomy was performed. After monitoring running-wheel activity and core temperature rhythms for approximately 2 mo, each animal was anesthetized and implanted with estradiol benzoate (EB). Crystalline EB (Sigma) was administered in $20 \mathrm{~mm}$ silastic capsules (Dow-Corning; $1.98 \mathrm{~mm} \mathrm{ID}$, $3.15 \mathrm{~mm} \mathrm{OD}$ ), filled to an effective length of $15 \mathrm{~mm}$ with hormone, and sealed at both ends with Medical Adhesive Silicone Type A (Dow-Corning). Capsules were implanted subcutaneously in the interscapular region (18). Vaginal opening and smears consistent with estrus were restored in eight of nine animals, and circadian activity and core temperature were examined in those eight animals. One animal's implant evidently failed to work since there was no vaginal opening following EB implantation. Data from this animal were not included in the analysis. After one week, estradiol capsules were removed.

Five animals were then given $1 \mathrm{wk}$ to recover from the effects of estradiol before being implanted with progesterone. As previously described for EB administration, crystalline progesterone (Sigma) was administered in $20 \mathrm{~mm}$ silastic capsules filled to an effective length of $15 \mathrm{~mm}$ with hormone, and implanted subcutaneously in the interscapular region. Capsules remained in place for $1 \mathrm{wk}$.

The daily mean, phase angle of entrainment, and amplitude of the circadian activity and core temperature rhythms were evaluated across four time periods: intact (non-estrus), postovariectomy (OVX), preimplant of each hormone, and postimplant of each hormone. Five to seven days representative of each period were selected for analysis as previously described. These data were also compared to estrous and metestrous data collected from these same animals while intact (Experiment 1).

Results

After ovariectomy, the cyclic effects of estrus on activity and core temperature were completely absent. Although animals 
TABLE 1

PHASE ANGLE OF ENTRAINMENT (Time in h)

\begin{tabular}{|c|c|c|c|c|c|}
\hline \multicolumn{6}{|l|}{ Intact Animals (Experiment 1) } \\
\hline $\begin{array}{l}\text { STAGE OF ESTRUS } \\
(N=10)\end{array}$ & $\Psi_{A B}$ & $\Psi_{\mathrm{TM}}$ & $\Psi_{\mathrm{A}}$ & $\Psi_{T p}$ & $\Psi_{T_{A}}$ \\
\hline PRE-ESTRUS & $+0.4 \pm 0.6^{\mathrm{a}}$ & $+2.3 \pm 0.3$ & $-1.9 \pm 1.0$ & $+0.2 \pm 0.1$ & $+2.0 \pm 0.5$ \\
\hline ESTRUS & $+2.3 \pm 0.3^{\mathrm{b}}$ & $+2.9 \pm 0.4$ & $-0.4 \pm 1.0^{\mathrm{c}}$ & $+1.3 \pm .4$ & $+0.4 \pm 0.5^{f}$ \\
\hline METESTRUS & $-2.6 \pm 1.0^{\circ}$ & $+2.2 \pm 0.7$ & $-3.9 \pm 2.9$ & $-2.2 \pm 1.2$ & $+4.3 \pm 1.4^{g}$ \\
\hline POST-ESTRUS & $-0.1 \pm 0.7^{\mathrm{d}}$ & $+2.2 \pm 0.5$ & $-3.9 \pm 1.3$ & $-0.9 \pm 0.8$ & $+2.4 \pm 0.5^{\mathrm{h}}$ \\
\hline \multicolumn{6}{|l|}{$\begin{array}{l}\text { Ovariectomized Animals } \\
\text { (Experiment 3) }\end{array}$} \\
\hline PRE-EB $(N=8)$ & $+1.3 \pm 0.1$ & $+2.5 \pm 0.2$ & $+0.3 \pm 0.1$ & $+0.1 \pm 0.1$ & $+1.2 \pm 0.1$ \\
\hline PLUS EB & $+2.2 \pm 0.8$ & $+3.6 \pm 0.4$ & $-0.4 \pm 0.7$ & $+1.0 \pm 0.4$ & $+1.3 \pm 0.6$ \\
\hline PRE-PROGEST $(N=5)$ & $-2.1 \pm 1.8$ & $+0.6 \pm 1.4$ & $-3.7 \pm 2.0$ & $-3.0 \pm 1.6$ & $+2.7 \pm 0.6$ \\
\hline PLUS PROGEST & $-4.1 \pm 1.6$ & $+1.0 \pm 0.8$ & $-5.3 \pm 1.7$ & $-1.7 \pm 1.3$ & $+5.2 \pm 1.4$ \\
\hline
\end{tabular}

${ }^{\mathrm{a}}$ Mean $\pm \mathrm{SEM} ;{ }^{\mathrm{h}} p<0.07$ from Pre-estrus; ${ }^{\mathrm{c}} p<0.001$ from Estrus, $p<0.003$ from Pre-estrus; ${ }^{\mathrm{d}} p<0.02$ from Metestrus, $p<0.002$ from Estrus; ${ }^{\mathrm{e}} p=0.055$ from Pre-estrus; ${ }^{\mathrm{i}} p<0.03$ from Pre-estrus; ${ }^{\mathrm{g}} p<0.005$ from Estrus, $p<0.045$ from Pre-estrus; ${ }^{\mathrm{h}} p<0.003$ from Estrus; ${ }^{\mathrm{i}} p<0.05$ from Pre-progest; $\Psi_{\mathrm{AO}}=$ Phase angle activity onset to lights on (LO); $\Psi_{\mathrm{AP}}=$ Phase angle morning activity peak to LO; $\Psi_{\mathrm{TM}}=$ Phase angle temperature minimum to LO; $\Psi_{\mathrm{TP}}=$ Phase angle moming temperature peak to LO; $\Psi_{\mathrm{TA}}=$ Phase angle between $\Psi_{\mathrm{TM}}$ and $\Psi_{\mathrm{AO}} ;$ EB $=$ Estradiol Benzoate; Progest $=$ Progesterone $($ crystalline); Pre-progest $=$ week following EB removal.

demonstrated a $16.9 \%$ decline in activity compared with the nonestrous period for intact animals, the decline was not significant (Fig. 6A). Also, no significant alterations were seen in $\Psi_{\mathrm{AO}}, \Psi_{\mathrm{AP}}$ or the amplitude of the circadian activity rhythm compared with the nonestrous state. In contrast, mean core temperature declined $.98^{\circ} \pm .07^{\circ} \mathrm{C}$ following ovariectomy (Fig. 6B; $p<0.02$ ). This alteration in mean temperature was sustained throughout the remainder of the study, despite the fact that animals continued to have access to running-wheels. There were no significant changes in $\Psi_{\mathrm{TM}}, \Psi_{\mathrm{TP}}$, or the amplitude of the temperature rhythm following ovariectomy.

Treatment with estradiol caused a nonsignificant increase in mean activity ( $35.7 \%$; Figs. 6A and 7A). There were no significant changes in $\Psi_{\mathrm{AO}}$ or $\Psi_{\mathrm{AP}}$ following estradiol implant (see Table 1, Fig. 7A), and activity amplitude did not differ significantly between intact, nonestrous or OVX states.

Estradiol did nor affect mean core temperature (Figs. 6B and 7B). As previously noted, there was a $.98^{\circ} \mathrm{C}$ decline in core temperature following ovariectomy, and this newly established mean temperature was not influenced by the estrogen implant. Phase angles of entrainment, $\Psi_{\mathrm{TM}}, \Psi_{\mathrm{TP}}$, and $\Psi_{\mathrm{TA}}$ were not altered by EB (Fig. 7, Table 1). The administration of estradiol was associated, however, with a decline in the amplitude of the temperature rhythm $(10.6 \% ; p<0.01)$ from the OVX, preEB condition.

One week following removal of the estradiol, progesterone was implanted. The degus demonstrated an immediate, decline in mean activity ( $54 \% ; p<0.001$, Fig. 6A) within the first $24 \mathrm{~h}$ of progesterone implantation, which continued throughout the remaining 4 days $(p<0.005$; Fig. $6 \mathrm{~A}) . \Psi_{\mathrm{AO}}, \Psi_{\mathrm{AP}}$, and activity amplitude were not significantly altered following progesterone treatment (Fig. 7A, Table 1).

Mean core temperature also declined $\left(.21^{\circ} \mathrm{C} ; p<0.005\right.$; Fig. $6 \mathrm{~B}$ ) following the administration of progesterone. $\Psi_{\mathrm{TM}}, \Psi_{\mathrm{TP}}$, and temperature amplitude did not differ significantly from the preprogesterone state (Fig. 7B, Table 1). However, $\Psi_{T A}$ was increased by progesterone treatment $(p<0.05$, Table 1$)$, as described for intact animals during metestrus (Experiment 1).

Therefore, as predicted, degus did not display cyclic alterations associated with estrus in the circadian activity and core temperature rhythms after ovariectomy. Although estradiol replacement reinstated vaginal estrus in these animals, it did not significantly increase either mean activity or core temperature. The only significant effect observed in EB-implanted animals was damping of the temperature rhythm amplitude. In contrast, progesterone replacement led to significant reductions in both mean activity and temperature. Neither hormone treatment significantly altered entrained phase angles of activity or temperature. However, after EB and progesterone treatments $\Psi_{\text {AO }}$ advanced or delayed $(p<.10)$ consistent with estrus or metestrus (Table 1). The delay in $\Psi_{A O}$ after progesterone resulted in a significant increase in $\Psi_{\mathrm{TA}}$ similar to that of metestrus.

\section{EXPERIMENT 4}

Experiments 1-3 demonstrate that estrus has a pronounced effect on the timing and expression of entrained circadian activity and core temperature rhythms in the diurnal degu. The daily mean and amplitude of both rhythms were altered during estrus, and the phase of activity onset was advanced. While it is possible that ovarian hormones act directly on the central circadian pacemaker, an alternative possibility is that gonadal hormones produce transient increases or decreases on mean activity and temperature with no direct impact on period length $(\tau)$. The short reproductive cycles of rats and hamsters (four to five days; 11 , 26) make it difficult to evaluate whether the effects of estrus on $\Psi_{\mathrm{Ao}}$ are the result of transient increases in mean activity, or if they reflect changes in the $\tau$. The longer estrous cycle of degus provides more time to determine whether hormonal changes alter $\tau$, or alter the duration of the active relative to the inactive phase of the circadian rhythm.

To further evaluate the influence of cyclic changes in steroids on the circadian pacemaker, seven intact female degus were placed in constant darkness (DD), and their free-running locomotor activity and core temperature rhythms were evaluated across the estrous cycle. Fourier and Chi square periodograms were used to determine the period of the activity and core temperature rhythms for 6-10 days before and after estrus. Using 

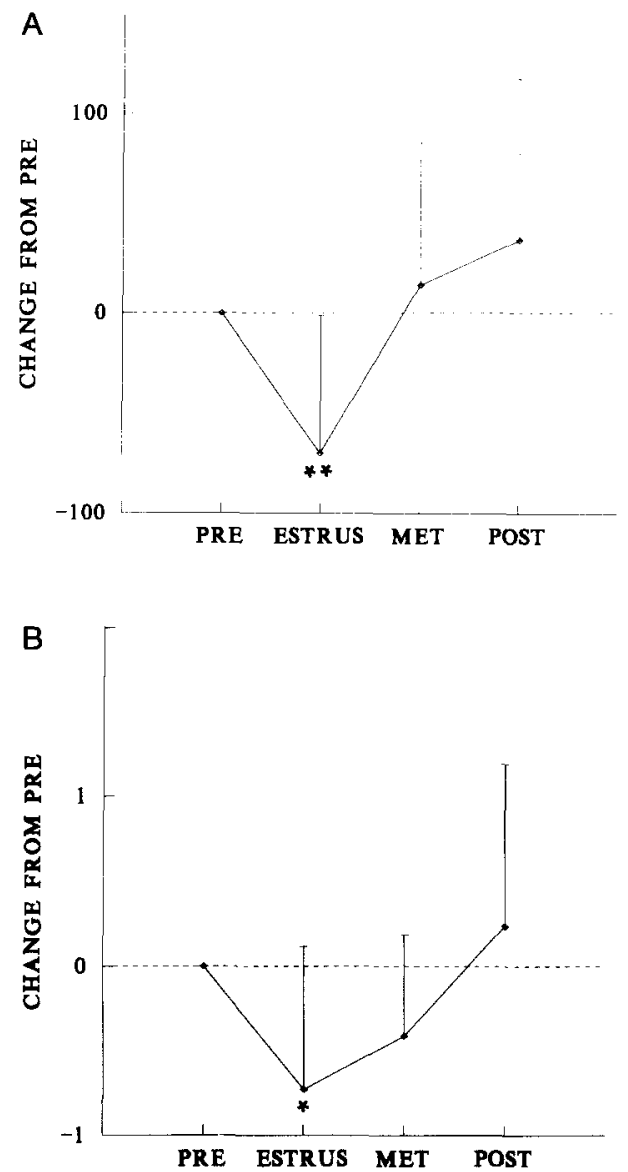

FIG. 4. Changes in rhythm amplitudes from preestrus (PRE) for circadian (A) running-wheel activity and (B) core temperature rhythms. Group designations as in Fig. $3\left(* p<0.05,{ }^{* *} p<0.01\right.$ with posthoc paired comparisons to preestrus).

the time of activity onset and temperature minimum as phase reference points, daily changes in $\tau$ were examined during preestrus, estrus, metestrus, and postestrus (see Experiment 1 for definitions). Postestrus included up to six days following metestrus to determine the stable postestrous $\tau$. The average, overall phase shift during estrus was calculated 4-6 days postestrus, and equalled the difference between the "actual" phase shift and the "predicted" phase shift based on preestrous $\tau$ derived from 710 days of data. Paired $t$-tests were used to examine actual vs. predicted changes in time of activity onset and temperature minimum on the day of estrus and metestrus.

\section{Results}

Thirteen estrous cycles (each animal contributing 1-3 estrous cycles to the data base) were used to examine the effect of estrus on the circadian activity and core temperature rhythms in DD. The average estrous period was $22.3 \pm 1$ days in DD, not significantly different from that in LD 12:12 $(p=0.063)$. In DD female degus displayed changes in activity and core temperature across the estrous cycle similar to those of intact animals in LD $12: 12$, although mean activity was somewhat depressed $(10 \%)$. Mean activity and core temperature rose on the day of estrus $\left(80 \%, p<0.02\right.$ and $.41^{\circ} \mathrm{C}, p<0.001$, respectively; Figs. 8 and 9). Metestrus was associated with a decline in mean activity
$(60 \%, p<0.008)$ and core temperature $\left(.56^{\circ} \mathrm{C}, p<0.001\right)$ compared with estrus (Figs. 8 and 9).

The average free-running $\tau$ was $23.7 \pm 0.09 \mathrm{~h}$ for both the activity and core temperature rhythms during the preestrous interval, and did not differ significantly during the postestrous interval $(23.8 \pm 0.08 \mathrm{~h})$. Compared to the average daily advance in the free-running rhythm, degus demonstrated a nonsignificant advance in activity onset on the day of estrus $(1.70+.66 \mathrm{~h}, p=$ $0.071)$, followed by a significant delay $(2.97 \pm 1.05 \mathrm{~h}, p<0.05)$ on the day of metestrus. However, the average overall phase shift in activity onset (determined 4-6 days after estrus) from the time predicted by preestrous $\tau$ was negligible $(0.013 \pm .137 \mathrm{~h}$; Fig. 8A). No significant changes from those predicted by preestrous $\tau$ were observed in the timing of daily temperature minimum or in $\Psi_{\mathrm{TA}}$ across the estrous cycle (Fig. 8B).

These data suggest that estrous-induced changes in ovarian hormones produce increases or decreases in mean temperature and activity, but only a transient effect on circadian phase of entrained animals. Alternatively, estrous-induced phase advances may be completely (and perfectly) reversed by metestrous delays.
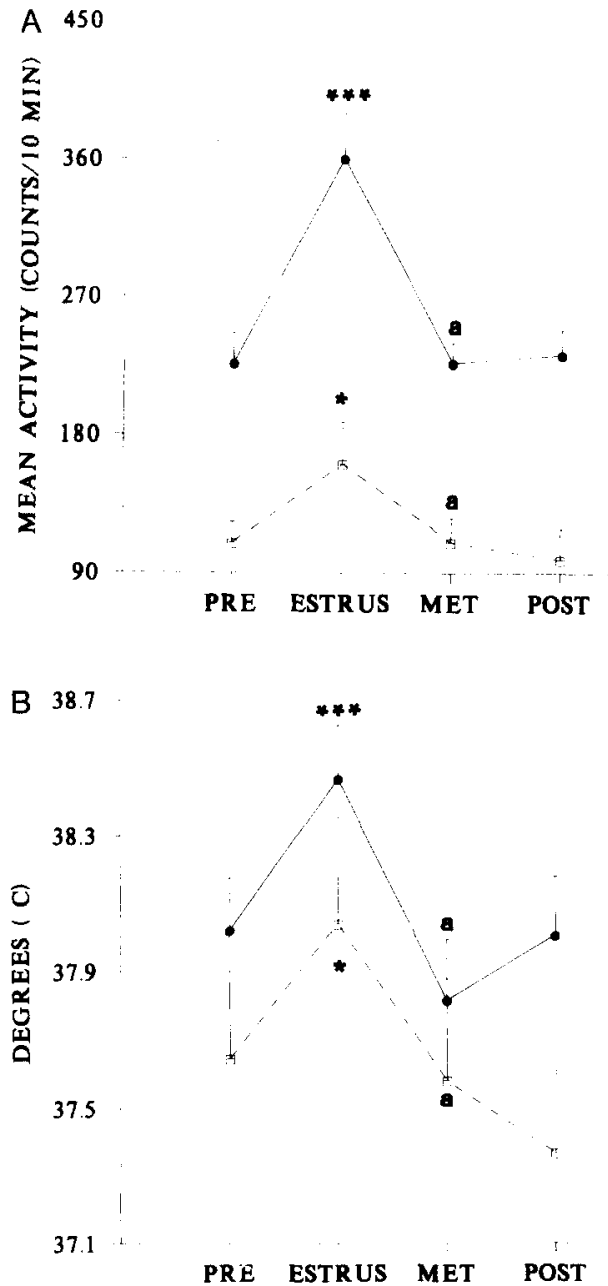

FIG. 5. Daily mean ( \pm SEM) (A) activity and (B) core temperature in female degus with $(\bullet)$ and without $(\square)$ running wheels across the estrous cycle. Group designations as in Fig. 3. Paired comparison differences from PRE are designated by asterisks $\left({ }^{*} p<0.05,{ }^{* * *} p<0.001\right)$. Paired comparison differences between estrus and metestrus are designated by " $a . "$ 
A

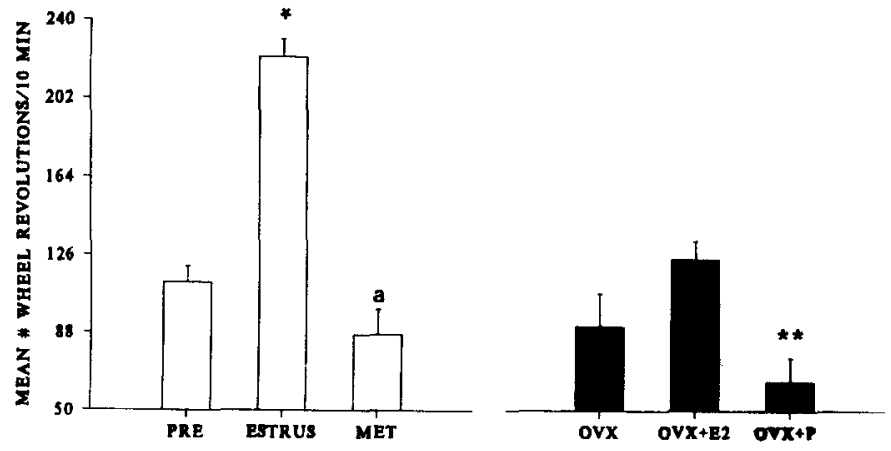

B

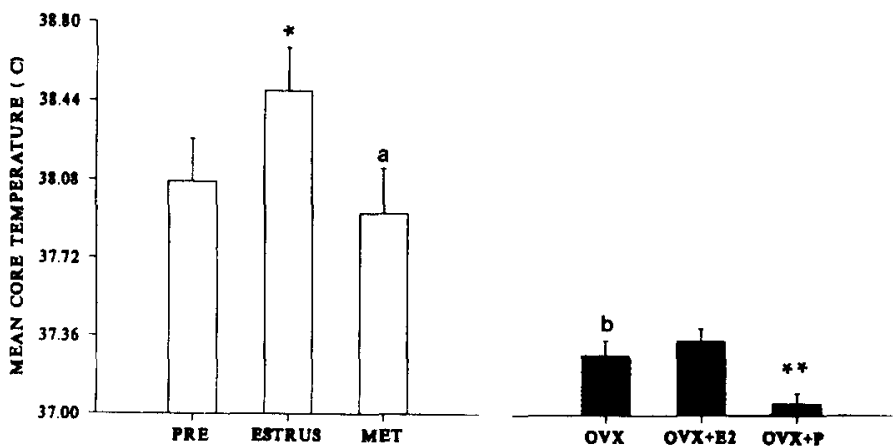

FIG. 6. Variation in mean daily (A) running-wheel activity and (B) core temperature before and after ovariectomy, estradiol implant, and progesterone implant. PRE, ESTRUS and MET as in Fig. 3, OVX = ovariectomized state, $\mathrm{OVX}+\mathrm{E} 2=$ plus estradiol implant, $\mathrm{OVX}+\mathrm{P}=$ plus progesterone implant. The same animals were used in each treatment; open bars $=$ intact, dark bars $=\mathrm{OVX}$. ${ }^{*}$ designates significant difference between PRE and ESTRUS, " $a$ " designates significant difference between ESTRUS and MET, " $b$ " designates significant difference between nonestrus-intact and OVX, $* *$ designates significant difference between $\mathrm{OVX}$ (after EB removal) and OVX $+\mathrm{P}$.

\section{EXPERIMENT 5}

This experiment evaluated the influence of estrogen and progesterone on free-running circadian activity and core temperature rhythms by first eliminating and then restoring the hormones. Five female degus, previously ovariectomized in Experiment 3, were housed in constant darkness (DD). After monitoring running-wheel activity and core temperature for 2 wk, each animal was anesthetized and implanted, under dim red light, with capsules containing estradiol benzoate (EB), as described in Experiment 3 . After one week capsules were removed, and animals were given 10 days to recover before being implanted with progesterone capsules (remained in place for $1 \mathrm{wk}$ ). The daily mean and amplitude of the circadian activity and core temperature rhythms were evaluated across four time periods: Preovariectomy (non-estrous days), postovariectomy, preimplant and postimplant. Fourier and Chisquare periodograms were used to determine activity and core temperature $\tau$ across these four periods.
Results

Following ovariectomy, female degus housed in DD demonstrated a $27.8 \%$ decline in mean activity $(p<0.02$, Fig. $9 \mathrm{~A})$ and a $.68^{\circ} \mathrm{C}$ decline in mean temperature $(p<0.008$, Fig. 9B) compared with free-running, intact, nonestrous animals. Estrous-related alterations in activity and core temperature were no longer apparent (Fig. 10).

Compared with the OVX state, mean activity $(55 \% ; p<$ 0.008 ; Figs. $9 \mathrm{~A}$ and $10 \mathrm{~A})$ and mean temperature $\left(.28^{\circ} \mathrm{C} ; p<\right.$ 0.025 ; Figs. $9 \mathrm{~B}$ and $10 \mathrm{~B}$ ) rose significantly after estradiol was implanted. Rhythm amplitudes were not altered by EB treatment.

Progesterone implantation brought about a $20 \%$ decline in mean activity from the week prior to implantation $(p<0.05$; Figs. 9A and 10A), however, there was no significant decline in mean temperature (Figs. 9B and 10B). As with EB, rhythm amplitudes were unaffected by hormone treatment.

While estradiol replacement significantly increased mean activity and core temperature and progesterone replacement signif- 


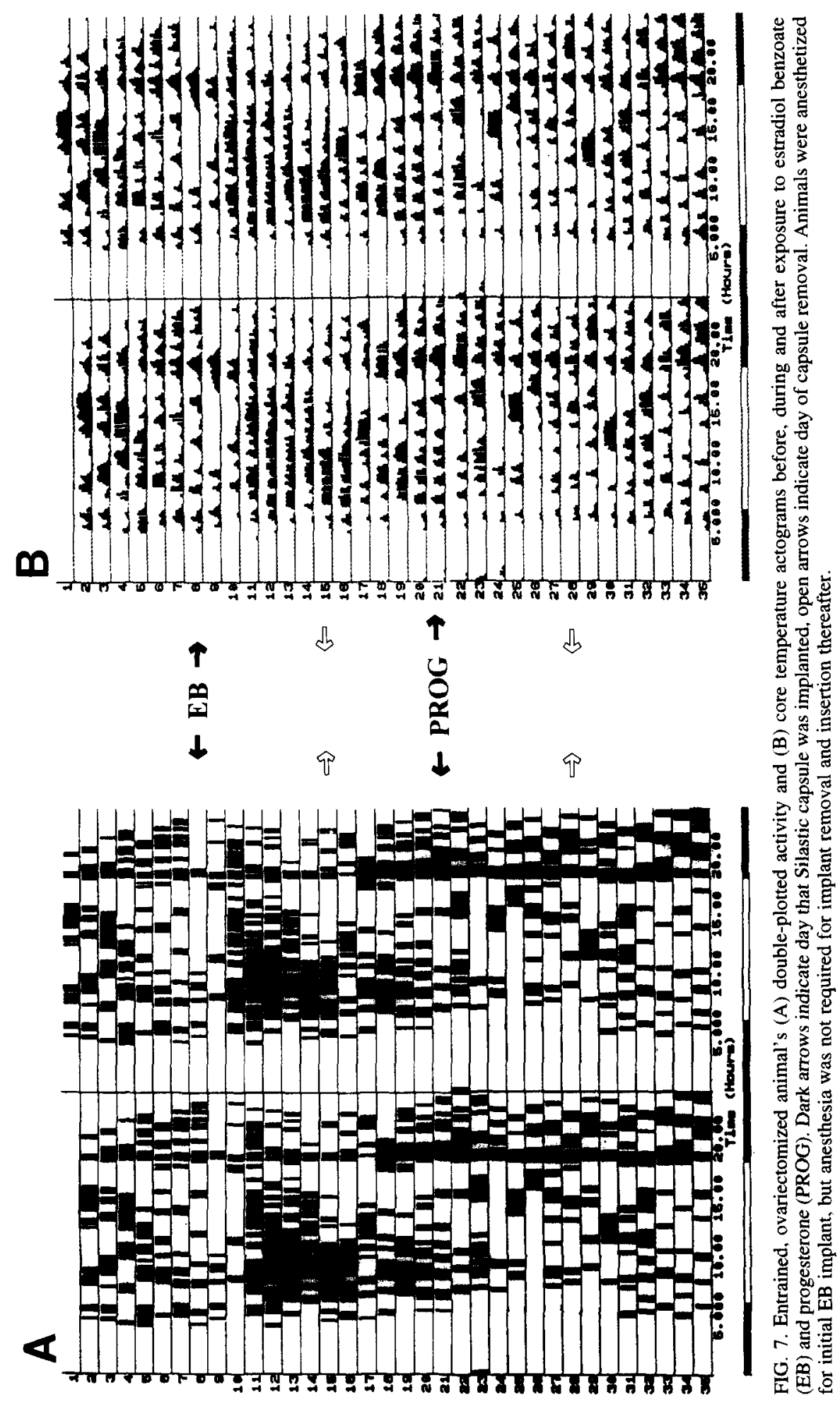


$\dot{\mathbf{A}}$

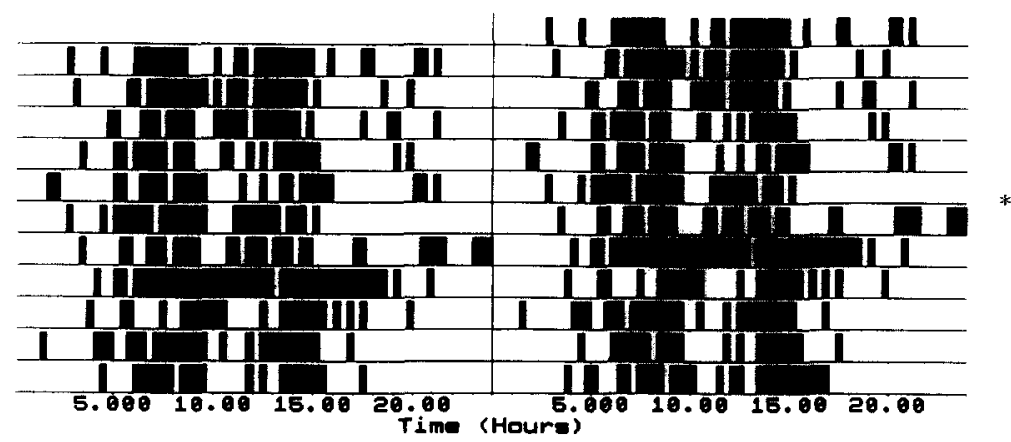

B

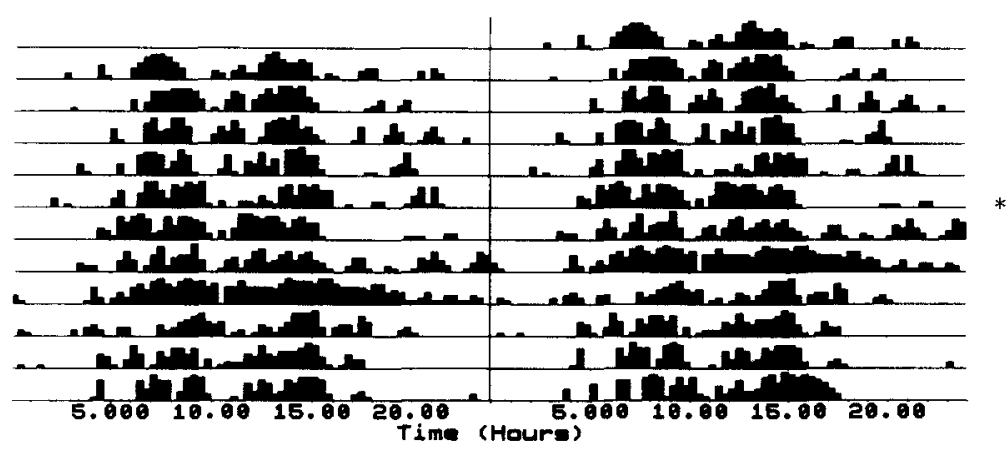

FIG. 8. Actogram of twelve days of double-plotted (A) wheel-running and (B) core temperature data for a single female degu maintained in constant darkness (DD). Asterisk denotes the time of estrus.

icantly decreased mean activity in OVX degus, the circadian activity and core temperature $\tau$ 's were not altered by either hormone treatment (Fig. 10).

\section{DISCUSSION}

These experiments support previous findings that circadian activity and core temperature rhythms are markedly altered during estrus in rodents. However, these experiments do not support the hypothesis that estrous-induced circadian changes in phase are the result of alterations in the period of the circadian clock. Rather, it seems more likely that alterations of entrained activity phase are the result of hormonally controlled alterations in the amount of locomotor behavior. Mean core temperature also is influenced by ovarian hormones, at times independent of effects those hormones have on activity.

On the day of estrus, entrained female degus demonstrated an increase in mean daily activity and core temperature, a phase advance in the circadian activity rhythm $\left(\Psi_{\mathrm{AO}}, \Psi_{\mathrm{AP}}\right)$, a reduction in the amplitude of both the circadian activity and core temperature rhythms, and a reduction in the phase angle difference between temperature minimum and activity onset $\left(\Psi_{T A}\right)$. On the day following estrus, $\Psi_{\mathrm{AO}}$ was phase delayed, the mean activity and core temperature fell below that of the preestrous period, and $\Psi_{\mathrm{TA}}$ was greatly increased (Experiment 1). These estrous-related changes in entrained circadian activity and core temperature in female degus are similar to those reported for other rodents. For example, female rats initiate activity earlier and display a significant increase in daily running-wheel activity and mean core temperature during proestrus/estrus $(1,15,20,26,35)$. Furthermore,
Kent et al. (15) noted that $50 \%$ of the female rats in their study demonstrated a consistent phase advance (at least $.5 \mathrm{~h}$ ) of the core temperature rhythm.

The rise in mean core temperature during proestrus in rats was positively correlated with the increase in activity, suggesting that the thermogenic response was induced by activity. Kent et al. (15) found that female Sprague-Dawley rats did not display significant elevations in mean core temperature during proestrus when running-wheels were locked or removed. In contrast, removing the running-wheels from degus did not alter estrous-related changes in core temperature (Experiment 2). While degus deprived of wheels demonstrated a decline in mean daily core temperature, it rose $.4^{\circ} \mathrm{C}$ on the day of estrus, exactly the same increase as when a wheel was present. Degus also demonstrated a decline in mean daily activity after wheel removal, however activity increased $40.4 \%$ on the day of estrus, similar to the increase of $54 \%$ with a wheel. Thus, removal of the running-wheel does not alter the degu's thermogenic response on the day of estrus, and there is only a weak association between temperature changes on the day of estrus and the absolute amount of activity.

As expected, ovariectomized degus did not demonstrate the estrous-related cyclic alterations in circadian activity and core temperature previously noted in intact animals. Furthermore, the mean core temperature declined significantly following ovariectomy and this decline persisted throughout the remainder of the experiments (Experiments 3 and 5). Similar reductions in mean core temperature following ovariectomy have been recorded in female rats (35) and rabbits (16), as well as in hypophysectomized mice (12). Furthermore, postmenopausal women do not display the postovulatory rise in mean core temperature seen in pre- 

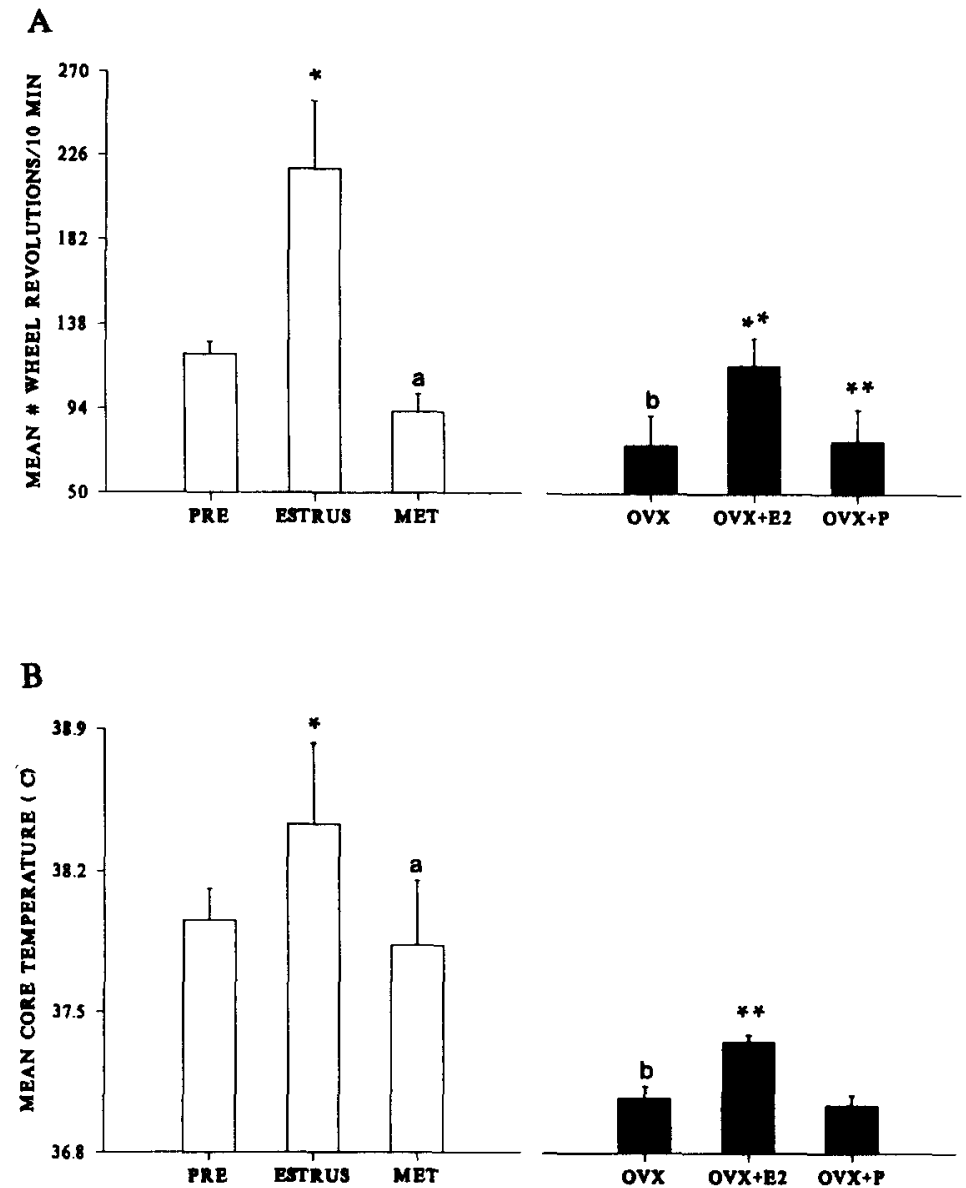

FIG. 9. Mean daily (A) running-wheel activity and (B) core temperature before and after ovariectomy, estradiol implant, and progesterone implant in female degus maintained in DD. Group and significance designations are as in Fig. 6, with ** now designating significant difference between OVX and OVX + hormone treatment.

menopausal women, and their mean core temperatures are permanently lowered (34). Results from these experiments indicate that core temperature of degus, but not mean activity, is sensitive to basal levels of ovarian hormones.

Ovariectomy of entrained females caused a nonsignificant decline in activity (Experiment 3 ). In contrast, OVX, free-running females (Experiment 5 ) displayed a significant $27.8 \%$ decline in activity with the decline in temperature. The apparently greater affect of OVX on activity of females in DD was likely the result of an additive influence of constant darkness (10\% decline in Experiment 4 compared with Experiment 1) and ovariectomy (16.9\% decline in Experiment 3 ) on activity, neither of which was significant alone. Core temperature of intact females housed in DD (Experiment 4) was exactly the same when compared with intact females in LD12:12 (Experiment 1), while OVX significantly decreased temperature (Experiment 3 ). We conclude that core temperature is influenced by basal ovarian hormones and not lighting conditions, while locomotor activity can be significantly altered only by decreasing both basal hormones and lights.

Estradiol replacement reinstated vaginal estrus in degus housed in LD12:12 and DD, but mean core temperature and activity were significantly elevated only in Experiment $5(p<.10$ for Experiment 3). However, in both experiments, EB implants produced far less of a response than did natural estrus. The lack of a significant phase advance in activity after EB implant might have been the result of depressed sensitivity to estrogen following long-term ovariectomy (19), compared with intact estrous females. In contrast, progesterone caused significant declines in mean temperature and activity for animals housed in LD12:12 and DD comparable to (or greater than) that of intact metestrus animals, yet $\Psi_{\mathrm{AO}}$ of entrained animals was still not significantly delayed. However, $\Psi_{T A}$ was significantly increased after progesterone, suggesting that the nonsignificant phase delay in $\Psi_{\mathrm{AO}}$ of entrained animals $(p<0.08)$ might have represented a small hormonal influence on the circadian clock. Thus, the results of Experiment 3 were inconclusive for determining whether steroid hormone changes during estrus were responsible for changes in $\Psi_{\text {AO }}$.

In these studies, metestrus or the administration of progesterone to ovariectomized degus resulted in a significant decline in mean activity and core temperature. This finding was surprising since progesterone has a thermogenic effect in women $(8,9,14)$. Women experience a rise in core temperature of $.5^{\circ}-1^{\circ} \mathrm{F}$ following ovulation. Pregnanediol, a progesterone metabolite, is thought to exert a thermogenic effect on thermoregulatory centers in the hypothalamus (29). A similar thermogenic response to progesterone has been demonstrated in rats $(13,23)$ and cattle (36). Following ovariectomy, female rats continued to display a ther- 


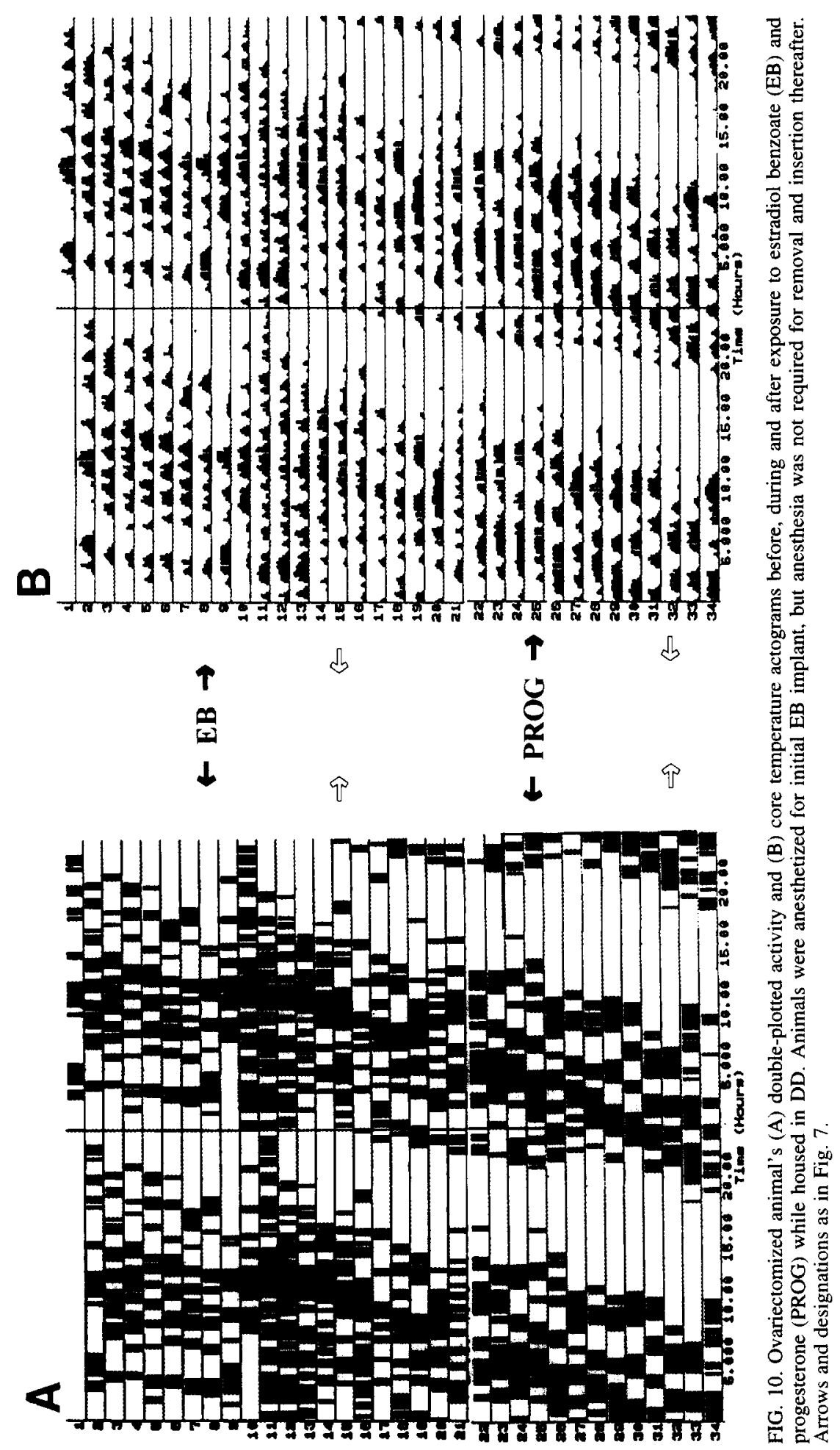


mogenic response to injections of progesterone, however, the hormone was less effective when compared to the response of intact animals (13). Why temperature responses to progesterone should differ in degus from other species so far studied is unclear, but the effect of progesterone or metestrus on temperature was consistent across all five experiments.

Although data from Experiments 2 and 3 indicate that temperature changes are not the result of changes in activity, the converse may be true. Significant changes in core temperature were accompanied by appropriate increases or decreases in mean activity and, for entrained cycling females, a phase change in activity onset. The one time this was not true, was maintenance of near normal levels of activity after ovariectomy caused core temperature to drop nearly $1^{\circ} \mathrm{C}$ (Experiment 3 ). However, from a given baseline core temperature, estrous-related changes in core temperature were associated with comparable changes in activity.

To further determine whether the estrous-induced changes in entrained circadian rhythms were the result of changes in period length, degus were maintained in DD while undergoing estrus (Experiment 4). Most animals in DD displayed transient fluctuations in the timing of daily activity onset and temperature minimum during, and immediately after estrus, however, the circadian period was not affected when examined 4-6 days after estrus. Ovariectomy eliminated all estrous-related fluctuations in activity and temperature, and subsequent treatment with estrogen and progesterone did not induce significant alterations in activity or core temperature $\tau$ 's (Experiment 5). Therefore, the estrous cycle and small hormone-induced changes in phase of entrained rhythms do not appear to be caused by hormonal effects on the circadian clock. Rather it is more likely that hormones act upon brain regions directly controlling locomotor activity (for review, 5 ) and temperature. Hormonally influenced activity control centers include the striatum $(6,27)$ and cerebellum $(30)$. Changes in phase of activity might then be likened to changes in levels of arousal to become more or less active, without changes in the clock.

The lack of changes in $\Psi_{\mathrm{AO}}$ or $\tau$ after ovariectomy or progesterone treatment caused significant declines in body temperature were surprising. Both theoretical (3) and empirical data suggest that changes in core temperature can cause alterations in $\Psi_{\mathrm{AO}}$ and/ or $\tau$ (e.g., 17,32,33). Thomas et al. (32) reported that even short periods of lowered core temperature during torpor, that were insufficient to significantly lower mean daily core temperature, were sufficient to significantly alter $\tau$ in Siberian hamsters. Perhaps degus have a $\mathrm{Q}_{10}$ closer to 1.0 than some other mammals, such that a larger change in core temperature than occurred in these experiments is required before an effect of change can be measured in $\Psi_{\mathrm{AO} O}$ or $\tau$.

Data on estrous cycle and hormone-induced changes in circadian period for hamsters might also be interpreted as having insignificant effects on the clock. The circadian activity period of free-running, intact, estrous hamsters advanced by about 20 min $(21,38)$, and the period shortened by $16 \mathrm{~min}$ in blind, ova- riectomized, estrogen-treated hamsters (31). In contrast to hamsters, $\tau$ was unchanged in degus following ovariectomy and treatment with steroids, and the nonsignificant transient change in phase on the day of estrus for degus in Experiment 4 was larger than that of hamsters. Hamsters demonstrate changes in activity level primarily at the onset of activity in response to endogenous or exogenous hormones (31). Increases and decreases in activity are not restricted to the onset of activity in degus, but are quite pronounced during that time. Data from both species suggest that ovarian hormones alter activity levels at the onset of the active period, causing changes in circadian phase, but have no permanent or major effect on $\tau$. This interpretation is supported by the lack of any significant change in temperature phase under any conditions for the degus.

In conclusion, we find cyclic changes in ovarian hormones have a pronounced effect on the timing and expression of the circadian activity and core temperature rhythms in the diurnal degu. Compared with rats and hamsters, the activity/temperature relationship has several unique characteristics: (i) access to running-wheels is not responsible for estrous-related circadian changes in core temperature; (ii) ovariectomy reduces mean temperature without a significant reduction of mean activity or change in phase for entrained animals, and without a change in $\tau$ for free-running animals; (iii) estrogen replacement in entrained, OVX degus brought about a nonsignificant increase in core temperature and activity, while progesterone replacement led to an immediate significant reduction in core temperature and mean activity, with neither hormone significantly altering $\Psi_{\mathrm{AO}}$; and (iv) in free-running conditions (DD), estrous-related fluctuations in the timing of daily activity onset and temperature minimum are transient, and there were no significant changes in $\tau$ with hormone treatment of OVX females. It is likely in the degus that hormonal affects on circadian activity and temperature do not directly involve the central circadian neural structures. Rather, hormone-induced changes in core temperature and, perhaps, also in brain circuits controlling locomotor activity, alter mean activity and activity phase in entrained, intact females during the estrous cycle.

\section{ACKNOWLEDGEMENTS}

We wish to thank Dr. Bill Xanten at the Washington National Zoo, John Gramieri at the Lincoln Park Zoo, Dave and Charlesetta Webster at the Scoville Children's Zoo, Dr. Milton Stetson at the University of Delaware, and Dr. Robert Davis at State University of N.Y. at Plattsburgh for their advice and assistance in establishing a breeding colony of $\boldsymbol{O}$. degus. We also wish to thank Dr. Philip Meyers and Dr. Barbara Lundrigan at the University of Michigan's Museum of Zoology for providing space for housing the colony; and two anonymous reviewers for their comments. This research was supported in part by NCNR grant NRO6397; NIMH grant MH49089; Wyeth-Ayerst Laboratories Fund of the American Nurses' Foundation; Rackham Graduate School, the Office of the Vice President of Research, and Continuing Education for Women at the University of Michigan; and Sigma Theta Tau (Rho Chapter).

\section{REFERENCES}

1. Albers, H. E.; Gerall, A. A.; Axelson, J. F. Effect of reproductive state on circadian periodicity in the rat. Physiol. Behav. 26:21-25; 1981.

2. Anantharaman-Barr, H. G.; Decombaz, J. The effect of wheel running and the estrous cycle on energy expenditure in female rats. Physiol. Behav. 46:259-263; 1989.

3. Aschoff, J. Circadian rhythms: Influences of internal and external factors on the period measured in constant conditions. Z. Tierpsychol. 49:225-249; 1979.
4. Baranczuk, R.; Greenwald, G. S. Peripheral levels of estrogen in the cyclic hamster. Endocrinology 92:805-812; 1973.

5. Becker, J. B. Hormonal influences on extrapyramidal sensorimotor function and hippocampal plasticity. In: Becker, J. B.; Breedlove, S. M.; Crews, D., eds. Behavioral endocrinology. Cambridge, MA: MIT Press; 1992:325-356.

6. Becker, J. B.; Cha, J. Estrous cycle-dependent variation in amphetamine-induced behaviors and striatal dopamine release assessed with microdialysis. Behav. Brain Res. 35:117-125; 1989. 
7. Binkley, S. Wrist activity in a woman: Daily, weekly, menstrual, lunar, annual cycles? Physiol. Behav. 52:411-421; 1992.

8. Cunningham, D. J.; Cabanac, M. Evidence from behavioral thermoregulatory responses of a shift in setpoint temperature related to the menstrual cycle. J. Physiol. 63:236-238; 1971.

9. Davis, M. E.; Fugo, N.W. Cause of physiological basal temperature changes in women. J. Clin. Endocr. 8:550-563; 1948.

10. Enright, J. T. Data analysis. In: Aschoff, J., ed. Handbook of behavioral neurobiology. vol 4: Biological rhythms. New York: Plenum Press; 1981:21-39.

11. Everett, J. W. Neurobiology of reproduction in the female rat: A fifty-year perspective. New York: Springer-Verlag; 1989.

12. Ferguson, D. J.; Visscher, M. B.; Halberg, F.; Levy, L. M. Effects of hypophysectomy on daily temperature variation in $\mathrm{C}^{3} \mathrm{H}$ mice. Am. J. Physiol. 190:235-238; 1957.

13. Freeman, N. E.; Crissman, J. K.; Jr.; Louw, G. N.; Butcher, R. L.; Innskeep, E. K. Thermogenic action of progesterone in the rat. Endocrinology 86:717-720; 1970.

14. Israel, S. L.; Schneller, O. The thermogenic property of progesterone. Fertil. Steril. 1:53-64; 1950.

15. Kent, S.; Hurd, M.; Satinoff, E. Interactions between body temperature and wheel running over the estrous cycle in rats. Physiol. Behav. 49:1079-1084; 1991

16. Kihlstrom, J. E.; Lundberg, C. Cyclic variation of body temperature in female rabbits before and after ovariectomy. Acta Physiol. Scand. $82: 272-276 ; 1971$.

17. Lee, T. M.; Holmes, W. G.; Zucker, I. Temperature dependence of circadian rhythms in golden-mantled ground squirrels. J. Biol. Rhythms 5:25-34; 1990.

18. Lee, T. M.; Zucker, I. Estradiol phase-shifts circannual rhythms of golden-mantled ground squirrels. Am. J. Physiol. 262:R1096R1099; 1992.

19. MacLusky, N. J.; Brown, T. J. Control of gonadal steroid receptor levels in the brain. In: Lakoski, J. M.; Perez-Polo J. R.; Rassin, D. K., eds. Neural control of reproductive function. New York: Alan R. Liss, Inc.; 1989:45-59.

20. Marrone, B. L.; Gentry, R. T.; Wade, G. N. Gonadal hormones and body temperature in rats: Effects of estrous cycles, castration and steroid replacement. Physiol. Behav. 17:419-425; 1976.

21. Morin, L. P.; Dark, J. Hormones and biological rhythms. In: Becker, J. B.; Breedlove, S. M.; Crews, D., eds. Behavioral endocrinology. Cambridge, MA: MIT Press; 1992:473-504.

22. Morin, L. P.; Fitzgerald, K. M.; Zucker, I. Estradiol shortens the period of hamster circadian rhythms. Science 196:305-307; 1977.

23. Nieburgs, H. E.; Greenblatt, R. B. The role of the endocrine glands in body temperature regulation. J. Clin. Endocrinol. 8:622-623; 1948.
24. Refinetti. R.; Menaker, M. Evidence for separate control of estrous and circadian periodicity in the golden hamster. Behav. Neural Biol. $58: 27-36 ; 1992$

25. Richards, M. P. Activity measured by running wheels and observation during the oestrous cycle, pregnancy and pseudopregnancy in the golden hamster. Anim. Behav. 14:450-458; 1966.

26. Richter C. P. Total self-regulatory functions. Harvey lectures. 38:63-103; 1942.

27. Roy, E. J.; Buyer, D. R.; Licari, V. A. Estradiol in the striatum: Effects on behavior and dopamine receptors but no evidence for membrane steroid receptors. Brain Res. Bull. 25, 221-227; 1990.

28. Ruiz de Elvira, M. C.; Persaud, R.; Coen, C. W. Use of running wheels regulates the effects of the ovaries on circadian rhythms. Physiol. Behav. 52:277-284; 1992.

29. Short, R. V. Oestrous and menstrual cycles. In: Austin, C. R.; Short, R. V., eds. Reproduction in mammals: vol. 3. Hormonal control of reproduction ( 2 nd ed.). Cambridge: Cambridge Univ. Press; 1984:115-152.

30. Smith, S. S.; Woodward, D. J.; Chapin, J. K. Sex steroids modulate motor-correlated increases in cerebellar discharge. Brain Res. 476:307-316; 1989

31. Takahashi, J. S.; Menaker, M. Interaction of estradiol and progesterone: Effects on circadian locomotor rhythm of female golden hamsters. Am. J. Physiol. 239:R497-R504; 1980.

32. Thomas, E. M.; Jewett, M. E.; Zucker, I. Torpor shortens the period of Siberian hamster circadian rhythms. Am. J. Physiol. 265:R951R956; 1993.

33. Tokura, H.; Aschoff, J. Effects of temperature on the circadian rhythm of pig-tailed macaques, Macaca nemestrina. Am. J. Physiol. 245:R800-R804; 1983.

34. Wever, R. A. Characteristics of circadian rhythms in human functions. J. Neur. Trans. 21(Suppl.):323-373; 1986.

35. Wollnik, F.; Turek, F. W. Estrous correlated modulations of circadian and ultradian wheel-running activity rhythms in LEW/ZTM rats. Physiol. Behav. 43:389-396; 1988.

36. Wrenn, T. R.; Bitman, J.; Sykes, J. The thermogenic influence of progesterone in ovariectomized cows. Endocrinology 65:317-321; 1959.

37. Yochim, J. M.; Spencer, F. Core temperature in the female rat: Effect of ovariectomy and induction of pseudopregnancy. Am. J. Physiol. $231: 361-365 ; 1976$

38. Zucker, I. Hormones and hamster circadian organization. In: Suda, M.; Hayaishi, O.; Nakagawa, $\mathrm{H}$., eds. Biological rhythms and their central mechanism. New York: Elsevier/North-Holland Biomedical Press; 1979:369-381. 